UNIVERSITY OF NOTTINGHAM

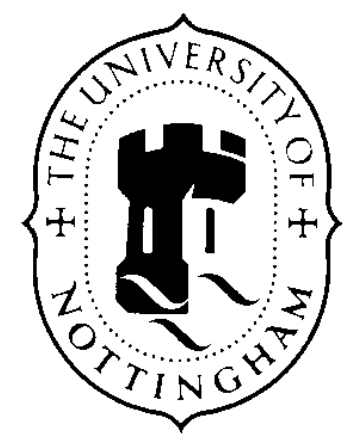

Discussion Papers in Economics

Discussion Paper

No. 07/06

Revealed Preference with Stochastic Demand

Correspondence

By Indraneel Dasgupta

June 2007 


\title{
Revealed Preference with Stochastic Demand Correspondence`
}

\author{
Indraneel Dasgupta \\ School of Economics, University of Nottingham, Nottingham NG7 2RD, UK. \\ E-mail: indraneel.dasgupta@nottingham.ac.uk \\ Prasanta K. Pattanaik ${ }^{\bullet}$ \\ Department.of Economics, University of California, Riverside, CA 92521, USA. \\ E-mail: prasanta.pattanaik@ucr.edu
}

This draft: 27 June 2007

\begin{abstract}
:
We unify and expand the theory of consumer's behavior, based on Samuelson's Weak Axiom of Revealed Preference, to permit simultaneously both random choice and non-singleton choice sets. We provide a consistency postulate for demand behavior when such behavior is represented in terms of a stochastic demand correspondence. When the consumer spends her entire wealth, our rationality postulate is equivalent to a condition we term stochastic substitutability. This equivalence generates: (i) Samuelson's Substitution Theorem, (ii) the central result in Bandyopadhyay, Dasgupta and Pattanaik (2004) and (iii) a version pertinent to deterministic demand correspondences (which independently yields Samuelson's Substitution Theorem), as alternative special cases. Relevant versions of the non-positivity of the own substitution effect, the demand theorem and homogeneity of degree zero in prices and wealth for the consumer's demand behavior, also follow as corollaries in every case.
\end{abstract}

Keywords: Stochastic demand correspondence, weak axiom of revealed preference, weak axiom of stochastic revealed preference, general substitution theorem, demand theorem.

JEL Classification Number: D11

- We thank M.M. Rao, Rahul Roy and seminar audiences at Cornell University, University of Edinburgh, University of California (Irvine), Indian Statistical Institute (Calcutta), Indian Statistical Institute (Delhi), University of Nottingham, University of California (Riverside) and University of York for helpful discussions. Dasgupta also acknowledges his intellectual debt to the late Dipak Banerjee, who introduced him to revealed preference theory.

• Corresponding author. Telephone: 1 (951) 827 1592; Fax: 1 (951) 8275685. 


\section{Introduction}

Arguably, the most fundamental finding in the classical revealed preference theory of consumers' demand concerns the inter-relationship between Samuelson's weak axiom of revealed preference (WARP) and various empirically testable restrictions on the demand behavior of a competitive consumer. The present paper expands this finding beyond the deterministic and single-element choice sets considered in the classical framework. Specifically, we construct a theory that simultaneously permits both probabilistic choice and non-singleton choice sets for consumers.

In a seminal paper, Samuelson (1938) introduced WARP. He deduced from it the conclusion that, if the consumption bundle chosen by a competitive consumer in some initial price-wealth situation costs exactly her wealth in some altered price-wealth situation, and differs from the bundle chosen in the new situation, then the products of price change and quantity change for each commodity must sum to a negative number. This conclusion, which we shall call Samuelson's Inequality (SI), summarizes most of the empirical content of the classical theory of consumers' behavior. SI implies non-negativity of the own-price substitution effect as a special case. This property, in turn, yields the familiar 'demand theorem' - other things remaining the same, a fall in the price of a normal good does not decrease the quantity of it purchased by the consumer. If the consumer always spends her entire wealth, SI also implies that demand functions must be homogeneous of degree zero in prices and wealth - changing all prices and wealth by the same proportion has no effect on the consumer's choice. Not only does WARP imply SI, but, for a consumer who always spends her entire wealth, SI also turns out to imply WARP (Samuelson (1948, 1947); Mas-Colell et al. (1995, pp. 28-32)). The equivalence of WARP and SI for a consumer who always exhausts her budget constitutes, arguably, the most fundamental result in the standard choicebased theory of consumers' behavior. For convenience, we shall term this equivalence Samuelson's Substitution Theorem.

The choice-based theory of consumer's behavior takes as its primitive some complete description of demand behavior for every possible price-wealth configuration. Its particular method of representation, however, imposes two major constraints at the very outset. First, faced with a given price-wealth situation, the consumer is assumed to choose a single consumption bundle. Thus, the consumer's demand behavior is constrained to representation by means of a demand function, rather than a demand correspondence. In consequence, the analytical framework cannot handle the choice counterpart of 'flat' indifference surfaces in the preference-based theory. Yet, it is not intuitively clear why, a priori, one should rule out the possibility that there are several consumption bundles in the consumer's budget set that she considers equally worthy of being consumed. ${ }^{1}$ Second, a

\footnotetext{
1 Observed market purchases would not reveal such multiplicity of 'optimal' consumption bundles for a given budget set since, by definition, the consumer can buy only one bundle from her budget set. One may, however, think of the problem of a consumer's choice in terms of consumer survey experiments, where consumers are asked to choose a (possibly multi-element) subset out of some feasible set of alternatives (e.g. Arrow (1959)).
} 
consumer's demand behavior is represented by means of a unique demand function. This assumes away the possibility of any probabilistic element in the consumer's choice. Yet, empirical evidence does often suggest the existence of a random aspect to individual decision-making; and a very large literature, with contributions by both economists and psychologists, argues the case for incorporating probabilistic choice within formal theoretical models. ${ }^{2}$ Modeling consumer's behavior by means of stochastic demand correspondences (SDCs), instead of the traditional deterministic demand functions (DDFs), would evidently allow one to accommodate these neglected aspects, thereby permitting a framework with greater flexibility and empirical scope.

The case for permitting multi-element choice sets was advocated early. Both Arrow (1959) and Sen (1971) specified versions of WARP applicable to general choice correspondences, though they did not address the specific issue of consumers' demand behavior. Richter (1966) explicitly developed the revealed preference theory of consumers' behavior in terms of demand correspondences, rather than demand functions. Afriat (1967) and Varian (1982) developed the generalized axiom of revealed preference (GARP) to accommodate 'flat' indifference surfaces. None of these contributions however addressed the issue of expanding the central result in the traditional theory, i.e., Samuelson's Substitution Theorem, to encompass demand correspondences. Their focus instead was on identifying restrictions that would allow demand behavior to be rationalized in terms of maximization on the basis of some complete and transitive binary preference relation, or even a utility function, requirements stronger than the satisfaction of WARP. ${ }^{3}$ It is not even clear from these contributions exactly how one should interpret either the non-positivity of the own-price substitution effect or the demand theorem, when the consumer's choice sets contain multiple consumption bundles. Furthermore, this literature restricted itself to deterministic choice behavior. Thus, the consumer's choice behavior was modeled in terms of a deterministic demand correspondence (DDC).

Additionally, as we discuss below, a consumer's observed market purchases may be consistently interpreted as choice on the basis of a demand correspondence, even when they violate Samuelson's WARP.

2 See, for example, Barbera and Pattanaik (1986), Block and Marschak (1960), Cohen (1980), Corbin and Marley (1974), Falmagne (1978), Fishburn (1973, 1977, 1978), Halldin (1974), Luce (1958, 1959, 1977), Luce and Suppes (1965), Manski (1977), Marschak (1960), McCausland (2007), McFadden (2005), Nandeibaum (2007), Quandt (1956), Sattath and Tversky (1976) and Yellott (1977). Econometricians routinely attempt to weigh probabilistic evidence against the predictions of a deterministic theory, devising statistical notions of 'goodness of fit' to accommodate the theory. This literature, in contrast, seeks to develop a theory that is itself probabilistic, so that it can be directly confronted with probabilistic evidence. Intuitively, observed randomness may come about either because preferences are inherently unstable, or because the external observer fails to notice changes in the environment that impact on the agent's decision-making.

${ }^{3}$ Houthakker (1950) developed the strong axiom of revealed preference (SARP) precisely to bridge this gap. GARP generalizes SARP to accommodate multi-element choice. Thus, much of the core empirical content of the standard theory of consumer's behavior, as summarized by SI, does not require rationalizability in terms of a transitive preference relation, just as much of the core empirical content of the traditional theory of a competitive firm's behavior does not entail rationalizability in terms of profit maximization (Dasgupta (2005)). Quah (2006) characterizes a class of complete but not necessarily transitive preference relations which yield demand functions satisfying WARP, and within which demand functions satisfying WARP can be rationalized. 
Pursuing a parallel, probabilistic, line of enquiry, Bandyopadhyay, Dasgupta and Pattanaik $(2004,1999)$ have recently presented a rationality postulate for stochastic demand behavior, the weak axiom of stochastic revealed preference (WASRP), and developed a stochastic version of Samuelson's Substitution Theorem. ${ }^{4}$ Their result encompasses the traditional version as a special case. However, their analysis was carried out in terms of a stochastic demand function (SDF). Thus, while Bandyopadhyay et al. (2004, 1999) departed from the classical framework by allowing the consumer to choose in a probabilistic fashion, they nevertheless constrained her to randomize only among alternative singleton sets of consumption bundles.

Approaches based on DDCs and SDFs extend the traditional DDF-based framework along different dimensions, both conceptually and empirically. From a conceptual, a priori perspective (e.g. Sen (1971), Richter (1966) and Arrow (1959)) it is obvious that probabilistic choice of a single consumption bundle and deterministic choice of multiple bundles are mutually exclusive a priori restrictions. Since random preferences and preferences that generate multiple best elements both appear independently plausible on a priori intuitive grounds, an attempt to develop a unified framework that simultaneously permits the choice counterparts of both these properties, by means of SDCs, may be motivated purely on such grounds.

One may however offer an alternative, a posteriori empirical motivation as well, directly in terms of making sense of a consumer's observed market purchases. First suppose a consumer is observed as choosing deterministically, in the sense of always choosing the same consumption bundle when faced with a given price-wealth situation, but violating WARP in its classical version. The theorist may then follow Afriat (1967) and Varian (1982) in setting herself the following task. Can she specify a DDC such that: (i) for every observed price-wealth configuration, the observed purchase belongs to the set of chosen bundles specified, and (ii) the DDC satisfies the relevant, expanded, version of WARP? If so, she can claim that, though observed choices falsify the behavioral hypothesis of the classical theory, they do not do so when the theory is expanded to permit multivalued choice, and market purchase is interpreted as picking a particular bundle out of the chosen set according to a particular behavioral rule. Suppose now the consumer's market purchases are observed repeatedly for two price-wealth situations, say 1 and 2 . In situation 1 , the consumer is observed to buy two bundles, $x^{*}$ and $y^{*}$, with identical frequency, while, in situation 2 , she is observed as picking two bundles, $x^{\prime}$ and $y^{\prime}$, with identical frequency. Evidently, such behavior is incompatible with the traditional DDF-based theory. Then, following the logic of the Afriat-Varian strategy outlined earlier, one can interpret observed market behavior in terms of a DDC, i.e., in terms of choice of some subsets $A^{*} \supseteq\left\{x^{*}, y^{*}\right\}, A^{\prime} \supseteq\left\{x^{\prime}, y^{\prime}\right\}$ in price-wealth situations 1 and 2, respectively. One

\footnotetext{
4 Bandyopadhyay et al. (2002) apply this postulate to demand aggregation, while Dasgupta and Pattanaik (2007) explore its connection with a weaker postulate, viz. regularity, in a general stochastic choice context.
} 
can also interpret such behavior in terms of an SDF that ascribes a probability of $\frac{1}{2}$ to $x^{*}$ and $x^{\prime}$ in situations 1 and 2, respectively. Representation in terms of an SDC is also possible. The methodological choice is of consequence. Under the assumption that the consumer exhausts her entire wealth, one can construct cases where observed choices will necessarily violate WASRP but not WARP for DDCs, and vice versa. Thus, the approaches based on SDFs and DDCs independently extend the empirical coverage of the theory. One can additionally construct cases that must violate both WASRP and WARP for DDCs, but are compatible with the underlying logic of either, when extended to allow simultaneously both random choice and choice of multiple consumption bundles. ${ }^{5}$ The existence of such situations implies that a theory based on a version of WARP that applies to SDCs offers the twin advantages of unification and expansion: while encompassing the existing versions as alternative special cases, such a theory can also accommodate forms of demand behavior that are incompatible with the existing versions.

Integration of the DDC-based and SDF-based strands of analysis would therefore appear to be of considerable interest, from an analytical, a priori perspective, as well as an a posteriori perspective of observed market demand. Furthermore, the extension to SDCs would also appear to mark the natural limits of the WARP-based approach to the analysis of consumer's behavior.

We provide such a unified and expanded theoretical framework. We develop a rationality, or consistency, postulate for demand behavior when such behavior is represented in terms of an SDC. Given that the consumer necessarily spends her entire wealth, our rationality postulate turns out to be equivalent to a condition we term stochastic substitutability. This equivalence generates the following as alternative special cases: (i) Samuelson's Substitution Theorem, (ii) the central result in Bandyopadhyay, Dasgupta and Pattanaik (2004), and (iii) a version pertinent to DDCs, which independently yields Samuelson's Substitution Theorem. Relevant versions of the non-positivity property of the own-price substitution effect, the demand theorem, and homogeneity of degree zero in prices and wealth for demand representations, all fall out as corollaries in every case.

Section 2 presents the basic notation. Section 3 develops the idea of representing demand behavior via stochastic demand correspondences. Section 4 defines some possible properties of stochastic demand correspondences. In particular, we define our notions of a normal good and the non-positivity property of the own substitution effect in this expanded context. We present and discuss our rationality postulate in Section 5. In this section, we also show how our rationality postulate, while necessarily encompassing other versions of the weak axiom as alternative special

\footnotetext{
5 We detail examples that establish these claims in Section 5. The DDC-based approach becomes compatible with every possible form of observed demand behavior if one does not assume wealth exhaustion (i.e., intuitively, the choice counterpart of 'thin' indifference surfaces): one can trivially rationalize anything in terms of a choice set that is always the entire budget set itself. Thus, WARP for DDCs is not empirically falsifiable, and, therefore, devoid of predictive consequence, even in principle, unless one assumes wealth exhaustion.
} 
cases, can additionally accommodate forms of demand behavior that violate such versions. Our results are presented in Section 6. Section 7 concludes. Proofs are presented in the appendix.

\section{Some Notation}

Let $m \geq 2$ be the number of commodities, and let $M=\{1,2, \ldots, m\}$ denote the set of commodities. $\mathfrak{R}_{+}, \mathfrak{R}_{++}$will denote, respectively, the set of all non-negative real numbers and the set of all positive real numbers. $\mathfrak{R}_{+}^{m}$ is the consumption set. The elements of the consumption set will be denoted by $x, x^{\prime}$ etc. Given any consumption bundle $x \in \mathfrak{R}_{+}^{m}$, and given any $i \in M, x_{i}$ will denote the amount of commodity $i$ contained in the bundle $x$.

The set of all possible price vectors is $\mathfrak{R}_{++}^{m}$, with $p, p^{\prime}$ etc. denoting individual price vectors. For any given commodity $i \in M$, we say that two price vectors $p$ and $p^{\prime}$ are $i$-variant iff [ $p_{i} \neq p_{i}^{\prime}$ and, for every commodity $j \neq i, p_{j}=p_{j}^{\prime}$ ]. The set of all possible wealth levels of the consumer is $\mathfrak{R}_{+}$, with $W, W^{\prime}$ etc. denoting specific wealth levels. A price-wealth situation is a pair $(p, W) \in \mathfrak{R}_{++}^{m} \times \mathfrak{R}_{+}$. Thus, the set of all possible price-wealth situations is $Z \equiv \mathfrak{R}_{++}^{m} \times \mathfrak{R}_{+}$. Given a

price-wealth situation $(p, W)$, the consumer's budget set, $B(p, W)$, is the set $\left\{x \in \mathfrak{R}_{+}^{m} \mid p \cdot x \leq W\right\}$. For brevity, we shall typically write $B, B^{\prime}$ etc., instead of $B(p, W), B\left(p^{\prime}, W^{\prime}\right)$, etc.

Given any non-empty set $T, r(T)$ will denote the set of all possible non-empty subsets of $T$ and $R(T)$ will denote the power set of $T$ (i.e., $R(T) \equiv r(T) \mathrm{U}\{\phi\}$, where $\phi$ denotes the empty set). Given two sets, $T$ and $T,\left[T \backslash T^{\prime}\right]$ will denote the set of all elements of $T$ that do not belong to $T^{\prime}$.

\section{Stochastic Demand Correspondence}

The first step in our analysis is to formalize the idea of modeling a consumer's demand behavior by means of a stochastic demand correspondence, and to locate this idea in relation to other possible, more traditional, representations. Given a price-wealth situation, the flexibility we seek intuitively involves allowing the consumer: (i) to have a choice set containing multiple consumption bundles (rather than a single bundle as in the classical framework), and (ii) to do so in a (non-trivially) random fashion (so that, in contrast to the classical framework, no single choice set is assigned probability 1).

\section{Definition 3.1.}

(i) A stochastic demand correspondence (SDC) is a rule $C$ which, for every $(p, W) \in Z$, specifies exactly one finitely additive probability measure $Q$ on $(r(B), R(r(B)))(r(B)$ being the set of outcomes and $R(r(B))$ being the relevant algebra in $r(B)$ ). 
(ii) A stochastic demand function (SDF) is a rule $D$ which, for every $(p, W) \in Z$, specifies exactly one finitely additive probability measure $q$ on $(B, R(B)$ ) ( $B$ being the set of outcomes and $R(B)$ being the relevant algebra in $B)$.

(iii) A deterministic demand correspondence (DDC) is a rule $c$ which, for every $(p, W) \in Z$, specifies exactly one non-empty subset of $B$.

(iv) A deterministic demand function (DDF) is a rule $d$ which, for every $(p, W) \in Z$, specifies exactly one element of $B$.

Consider any price-wealth situation $(p, W)$. Let $B$ denote the budget set corresponding to $(p, W)$. Let $Q=C(p, W)$, where $C$ is an SDC. Given the price-wealth situation $(p, W)$, for any $A \in R(r(B)), Q(A)$ is the probability that the (possibly multi-element) set of chosen bundles will lie in the class $A$. $C(p, W), C\left(p^{\prime}, W^{\prime}\right)$ etc. will be denoted, respectively, by $Q, Q^{\prime}$ etc. Thus, an SDC captures the idea that, given a budget set: (i) the consumer may choose a subset with multiple elements, and (ii) she may choose among the alternative subsets available in a probabilistic fashion. An SDF, introduced by Bandyopadhyay, Dasgupta and Pattanaik (1999), restricts the consumer to choosing a single consumption bundle, albeit allowing her to do so in a probabilistic fashion. Let $q=D(p, W)$, where $D$ is an SDF. For any $A \in R(B), q(A)$ is the probability that the chosen bundle will lie in the set $A$. Thus, the intuitive idea captured by an SDF is that the consumer always chooses only one consumption bundle; however, exactly which bundle is going to be chosen is determined by some probabilistic rule. A DDC, in contrast, assumes away the probabilistic element in choice, but allows the possibility that the consumer will choose multiple bundles. Thus, given a DDC, $c, c(p, W) \in r(B)$ is the (possibly multi-element) set of consumption bundles the consumer will choose from the budget set corresponding to $(p, W)$. A DDF is the most restrictive, yet also the most common, form of representation of demand behavior in consumer theory. This constrains the consumer's choice from a budget set to a single consumption bundle, chosen according to some deterministic (i.e. non-probabilistic) decision rule. Given a DDF, $d, d(p, W) \in B$ is the bundle the consumer will choose from the budget set corresponding to the price-wealth situation $(p, W)$.

It is evident from the preceding discussion that an SDC is the most flexible tool available for modeling a consumer's demand behavior. Intuitively, SDFs, DDCs and DDFs are all special classes of SDCs. We now proceed to provide a formal statement of this idea. 


\section{Definition 3.2.}

(i) An SDC, $C$, is singular, iff, for all $(p, W) \in Z$, and for every $A \in R(r(B))$, $Q(A)=Q\left(\left\{A_{i} \in A|| A_{i} \mid=1\right\}\right)$.

(ii) An SDC, $C$, is degenerate, iff, for all $(p, W) \in Z$, there exists $A \in r(B)$ such that $Q(\{A\})=1$.

A singular SDC is one where the consumer's probability of choosing a set with multiple consumption bundles is zero. A degenerate SDC is one where the consumer chooses, in effect, in a deterministic fashion. A singular SDC corresponds to an SDF, a degenerate SDC corresponds to a DDC, and a singular and degenerate SDC corresponds to a DDF. More formally, we define the following.

\section{Definition 3.3.}

(i) An SDC, $C$, induces an SDF, $D$, iff, for every $(p, W) \in Z$, and for every $A \in R(B)$, $q(A)=Q(\{\{x\} \mid x \in A\})$. An SDF, $D$, induces an SDC, $C$, iff, for every $(p, W) \in Z$, and for every $A \in R(r(B)), Q(A)=q(\{x \in B \mid\{x\} \in A\})$.

(ii) An SDC, $C$, induces a DDC, $c$, iff, for every $(p, W) \in Z, c(p, W)$ is the set of consumption bundles $A \subseteq B$ such that $Q(\{A\})=1$. A DDC, $c$, induces an SDC, $C$, iff, for every $(p, W) \in Z$, $Q(\{c(p, W)\})=1$

Remark 3.4. Let $C$ be a non-singular SDC and let $D$ be an SDF. Given that $C$ is non-singular, we must have $Q(\{\{x\} \mid x \in B\})<1$; yet it must always be the case that $q(B)=1$. Therefore, $C$ cannot possibly induce $D$. Thus, only singular SDCs can induce SDFs. Now notice that, an SDC that is induced by an SDF must satisfy $Q(\{\{x\} \mid x \in B\})=1$; thus, every SDC induced by an SDF must be singular. Notice further that every singular SDC induces some SDF, and every SDF induces some singular SDC. If a singular SDC, $C$, induces some SDF, $D$, then the singular SDC induced by $D$ must be $C$ itself. Analogous relations can easily be seen to hold between degenerate SDCs and DDCs, and also between DDFs and SDCs that are both singular and degenerate.

Notice now a methodological difference between the notions of DDF and SDF on the one hand and those of DDC and SDC on the other. The concept of choice involved in a DDF and an SDF can be interpreted in terms of the consumer's actual purchase of a consumption bundle in the market place. The difference is that, in the case of a DDF, the consumer always purchases the same consumption bundle from a given budget set, while, in the case of an SDF, the consumer's purchase can be stochastic in nature. In contrast, both DDCs and SDCs permit multi-element choice sets, 
which cannot be directly observed by watching the consumer's actual market purchases: the consumer cannot simultaneously buy multiple consumption bundles from her budget set, even if she chooses multiple ones. This implies that axioms regarding the consumer's behavior, which permit multielement choice sets, are not directly testable when we confine ourselves to observations of actual market behavior. If, however, one does not confine oneself to market choices, it may be possible to 'observe' a multi-element choice set. For example, in response to a questionnaire, the consumer may indicate that, given the price-wealth situation, she does not mind choosing either bundle $x$ or bundle $y$, but will reject every other consumption bundle in her budget set.

Of course, a theoretical structure, constructed in terms of either a DDC or an SDC, can be used to derive conclusions about the consumer's market behavior. In that case, though the observation of the consumer's market choices cannot be used to test the assumptions of the theory directly, such observations can be used to test the assumptions indirectly by testing the conclusions. This will require some assumption to link the consumer's observed market behavior to his possibly multi-element choice set. The weakest possible assumption is obviously the rule that the purchased bundle must be a member of the realized choice set. As we shall argue later, though we use the notion of an SDC as our primitive concept, our results have obvious intuitive interpretation in terms of the observed market behavior of the consumer, even under this weakest conceivable assumption regarding the link between multi-element choice sets and the consumer's observed market behavior.

\section{Some properties of SDCs}

We now formulate some properties that an SDC may conceivably have. Our substantive results, presented in Section 6, will involve identifying the interconnections between these properties and a behavioral postulate that we shall introduce in Section 5 .

Definition 4.1. An SDC, $C$, is tight iff, for every $(p, W) \in Z, Q\left(r\left(\left\{x \in \mathfrak{R}_{+}^{m} \mid p \cdot x=W\right\}\right)\right)=1$. An SDF is tight iff the singular SDC induced by it is tight. Tightness for DDCs and DDFs is defined analogously.

Tightness is the intuitively appealing requirement that the probability of the consumer's having a choice set, such that every consumption bundle in the choice set exhausts her entire wealth, is always one. In other words, the consumer rejects all consumption bundles that do not exhaust her wealth. In essence, this is the choice counterpart of the standard global non-satiation presumption. We shall derive our substantive results in Section 6 under the assumption that the SDC satisfies this restriction.

We proceed to define the requirement, familiar from the traditional theory, that demand behavior should not change when all prices and wealth change in the same proportion, so that the 
consumer's budget set remains unaltered. Evidently, in our expanded context, this property translates into the requirement that her probability of choosing from any given collection of subsets of her budget set should not change, so long as all prices and wealth change by the same proportion.

Definition 4.2. An SDC, $C$ satisfies homogeneity of degree zero in all prices and wealth $(\mathrm{H}(0))$ iff, for all $(p, W),\left(p^{\prime}, W^{\prime}\right) \in Z$ such that $\left[p^{\prime}=\lambda p, W^{\prime}=\lambda W\right.$ for some $\left.\lambda \in \mathfrak{R}_{++}\right], Q=Q^{\prime}{ }^{6}$ An SDF satisfies $\mathrm{H}(0)$ iff the singular SDC induced by it is $\mathrm{H}(0)$. $\mathrm{H}(0)$ for DDCs and DDFs is defined analogously.

Our next step is to reinterpret the standard notion of non-positivity of the own-price substitution effect. In the traditional, DDF-based framework, if the price of only one commodity, say $i$, falls, all other prices remaining constant, and the consumer's wealth is compensated so that she can exactly afford the old consumption bundle, this property requires that the amount of the $i$-th commodity demanded 'should not fall'. In articulating this idea for an SDC, one faces two intuitive difficulties. First, since the consumer may now choose multiple bundles initially, which one should be our reference bundle for determining the level of compensation? Second, since she may now choose multiple amounts of the $i$-th commodity in both situations, how should we interpret the idea of demand 'not falling', compared to the initial situation? Our answer to the first problem is to consider every consumption bundle that the consumer could possibly have chosen initially as a reference bundle for determining the level of compensation. Our answer to the second is to interpret the idea of demand for the $i$-th commodity 'not falling' in a probabilistic set-dominance sense, in relation to the amount contained in this initial (arbitrary) reference bundle.

Consider therefore the following scenario. Suppose, starting from some initial price-wealth situation $(p, W)$, the price of commodity $i$ falls from $p_{i}$ to $p_{i}^{\prime}$, all other prices remaining invariant. Consider any arbitrary amount of the $i$-th commodity, $\alpha$, that the consumer could possibly have bought in the initial situation. The price fall reduces the cost of every consumption bundle containing this amount of the $i$-th commodity by $\left(p_{i}-p_{i}^{\prime}\right) \alpha$. Suppose now that the consumer's wealth is exactly compensated for this cost reduction. Thus, suppose that the consumer's wealth is reduced by exactly $\left(p_{i}-p_{i}^{\prime}\right) \alpha$, so that $W^{\prime}=W-\left(p_{i}-p_{i}^{\prime}\right) \alpha$. Then all consumption bundles containing at least $\alpha$ amount of the $i$-th commodity that were initially available continue to be available in the new price-wealth situation. Furthermore, the consumer can now afford some consumption bundles containing more than $\alpha$ amount of the $i$-th commodity, that she initially could not. However, some consumption bundles containing less than $\alpha$ amount of the $i$-th commodity, which were initially available, now become unaffordable. How would the consumer respond to the new price-wealth

\footnotetext{
${ }^{6}$ Notice that, by construction, $B=B^{\prime}$.
} 
situation? More specifically, what is the probability that the consumer will choose, in the new situation, a set of consumption bundles where every bundle contains at least $\alpha$ of the $i$-th commodity? Suppose that this probability is not less than the initial probability of choosing a set of consumption bundles where at least one consumption bundle contains at least $\alpha$ of the $i$-th commodity. Suppose further that an analogous relationship holds for sets of consumption bundles containing more than $\alpha$ of the $i$-th commodity. We shall then say that the SDC satisfies nonpositivity of the own substitution effect.

Definition 4.3. A tight SDC, $C$, satisfies non-positivity of the own substitution effect (NPS) iff, for every $i \in M$, and for all $(p, W),\left(p^{\prime}, W^{\prime}\right) \in Z$ such that $\left[p, p^{\prime}\right.$ are $i$-variant with $p_{i}^{\prime}<p_{i}$, and

$$
\begin{aligned}
\left.\alpha \equiv\left(\frac{W-W^{\prime}}{p_{i}-p_{i}^{\prime}}\right) \in\left[0, \frac{W}{p_{i}}\right]\right], \\
Q^{\prime}\left(\left\{s^{\prime} \subseteq B^{\prime} \mid x_{i}^{\prime}>\alpha \text { for all } x^{\prime} \in s^{\prime}\right\}\right) \geq Q\left(\left\{s \subseteq B \mid x_{i}>\alpha \text { for some } x \in s\right\}\right),
\end{aligned}
$$

and

$$
Q^{\prime}\left(\left\{s^{\prime} \subseteq B^{\prime} \mid x_{i}^{\prime} \geq \alpha \text { for all } x^{\prime} \in s^{\prime}\right\}\right) \geq Q\left(\left\{s \subseteq B \mid x_{i} \geq \alpha \text { for some } x \in s\right\}\right) \text {. }
$$

Next, we expand the notion of a normal good from its classical context. As before, we face the problem of interpreting the idea of demand 'not falling', but now in response to an increase in the consumer's wealth: we utilize an analogous probabilistic set-dominance criterion to do so. Suppose a consumer's wealth increases, while all prices remain constant. Consider any arbitrary amount of the $i$-th commodity, $\alpha$. What is the probability that the consumer will choose, in the new situation, a set of consumption bundles where every bundle contains at least $\alpha$ of the $i$-th commodity? Suppose this probability is not less than the corresponding probability in the initial situation. Suppose also that an analogous restriction holds for sets of consumption bundles containing more than $\alpha$ of the $i$-th commodity. We shall then call the $i$-th commodity a normal good.

Definition 4.4. Given an SDC, $C$, a commodity, $i$, is normal, iff, for all $\alpha \in \mathfrak{R}_{+}$, all $p \in \mathfrak{R}_{++}^{m}$, and all $W, W^{\prime} \in \mathfrak{R}_{+}$such that $W^{\prime}>W$,

$$
Q^{\prime}\left(\left\{s^{\prime} \subseteq B^{\prime} \mid x_{i}^{\prime}>\alpha \text { for all } x^{\prime} \in s^{\prime}\right\}\right) \geq Q\left(\left\{s \subseteq B \mid x_{i}>\alpha \text { for all } x \in s\right\}\right),
$$

and

$$
Q^{\prime}\left(\left\{s^{\prime} \subseteq B^{\prime} \mid x_{i}^{\prime} \geq \alpha \text { for all } x^{\prime} \in s^{\prime}\right\}\right) \geq Q\left(\left\{s \subseteq B \mid x_{i} \geq \alpha \text { for all } x \in s\right\}\right)
$$

where $B=B(p, W)$ and $B^{\prime}=B\left(p, W^{\prime}\right){ }^{7}$

\footnotetext{
${ }^{7}$ Notice that (4.1') and (4.2') above are weaker analogues of (4.1) and (4.2), respectively.
} 
We shall call a good regular if, intuitively, its demand does not fall with a fall in its own price, the consumer's wealth and all other prices remaining invariant. As before, we shall use a probabilistic set-dominance criterion to formalize the notion of demand 'not falling'. Thus, in our subsequent analysis, the familiar demand theorem will simply constitute the claim that every normal good is also regular. ${ }^{8}$

Definition 4.5. Given an SDC, $C$, a commodity, $i$, is regular iff, for all $p, p^{\prime} \in \mathfrak{R}_{++}^{m}$ such that [ $p, p^{\prime}$ are $i$-variant with $p_{i}^{\prime}<p_{i}$ ], for every $W \in \mathfrak{R}_{+}$, and for all $\alpha \in \mathfrak{R}_{+}$, (4.1) and (4.2) both hold when $B=B(p, W)$ and $B^{\prime}=B\left(p^{\prime}, W\right)$.

Lastly, we define a restriction for SDCs when more than one, possibly all, prices are allowed to change simultaneously. This condition is essentially an expansion of a condition introduced by Bandyopadhyay, Dasgupta and Pattanaik (2004) in the context of SDFs, which in turn constituted an expansion of Samuelson's Inequality from its classical, DDF context. Following their terminology, we call our condition stochastic substitutability.

Notation 4.6. Given two price-wealth situations $(p, W),\left(p^{\prime}, W^{\prime}\right)$, let:

$$
\begin{aligned}
& I=\left\{x \in \Re_{+}^{m} \mid p \cdot x=W \text { and } p^{\prime} \cdot x=W^{\prime}\right\}, \\
& G=\left\{x \in \Re_{+}^{m} \mid p \cdot x=W \text { and } p^{\prime} \cdot x>W^{\prime}\right\}, \\
& H=\left\{x \in \mathfrak{R}_{+}^{m} \mid p \cdot x=W \text { and } p^{\prime} \cdot x<W^{\prime}\right\}, \\
& G^{\prime}=\left\{x \in \mathfrak{R}_{+}^{m} \mid p \cdot x>W \text { and } p^{\prime} \cdot x=W^{\prime}\right\},
\end{aligned}
$$

and

$$
H^{\prime}=\left\{x \in \Re_{+}^{m} \mid p \cdot x<W \text { and } p^{\prime} \cdot x=W^{\prime}\right\} .
$$

The various sets defined in Notation 4.6 are illustrated in Figure 1 for the two-commodity case.

\section{Insert Figure 1}

\footnotetext{
8 We could alternatively impose a stronger notion of normal and regular goods by requiring the relevant inequalities to hold strictly for Definitions 4.4 and 4.5, so that, intuitively, demand is required to 'rise', rather than 'not fall', with, respectively, a rise in wealth and a fall in own price.
} 
Definition 4.7. A tight SDC, $C$, satisfies stochastic substitutability (SS) iff, for every ordered pair $\left\langle(p, W),\left(p^{\prime}, W^{\prime}\right)\right\rangle \in Z^{2}$, and for every $A \subseteq I$,

$$
\begin{aligned}
& Q^{\prime}\left(r\left(G^{\prime}\right)\right)+Q^{\prime}\left(\left\{s^{\prime} \subseteq\left(G^{\prime} \mathrm{Y} I\right) \mid \phi \neq\left(s^{\prime} \mathrm{I} I\right) \subseteq A\right\}\right) \geq \\
& Q(\{s \subseteq B \mid \phi \neq(s \text { I I }) \subseteq A\})+Q(\{s \subseteq B \mid(s \text { I I })=\phi,(s \text { I } H) \neq \phi\}) .
\end{aligned}
$$

Suppose, from some initial price-wealth configuration $(p, W)$, there is a shift to some new configuration $\left(p^{\prime}, W^{\prime}\right)$. Let $\bar{X}$ be some consumption bundle that costs the consumer's entire wealth in both price-wealth situations (so that $p^{\prime} \cdot \bar{x}=W^{\prime}$ and $p \cdot \bar{x}=W$ ). Consider any collection, $A$, of such bundles. The LHS of the inequality in (4.3) is the probability that the consumer will choose a set $s^{\prime}$ in the new situation such that: (i) $\left(p-p^{\prime}\right)\left(\bar{x}-x^{\prime}\right) \leq 0$ for every bundle $x^{\prime}$ in $s^{\prime}$, and (ii) whenever $\left(p-p^{\prime}\right)\left(\bar{x}-x^{\prime}\right)=0$ for some bundle $x^{\prime}$ in $s^{\prime}$, that bundle $x^{\prime}$ also lies in A. The RHS of the inequality is the probability that the consumer chose a set $s$ in the initial situation such that: (i) $\left(p-p^{\prime}\right)(\bar{x}-x) \leq 0$ for at least one bundle $x$ in $s$, and (ii) whenever $\left(p-p^{\prime}\right)(\bar{x}-x)=0$ for some bundle $x$ in $S$, that bundle $x$ also lies in A. SS requires the first to be no less than the second.

\section{Rationality postulate}

The last major building block for our substantive analysis in Section 6 is also the central element. This is a rationality, or consistency, postulate for demand behavior. We now introduce this postulate.

Definition 5.1. An SDC, $C$, satisfies the weak axiom of stochastic revealed preference (WASRP) iff, for every ordered pair $\left\langle(p, W),\left(p^{\prime}, W^{\prime}\right)\right\rangle \in Z^{2}$, and for every $A \in r\left(B\right.$ I $\left.B^{\prime}\right)$,

$$
Q\left(r\left(B \backslash B^{\prime}\right)\right) \geq Q^{\prime}\left(\left\{s^{\prime} \subseteq B^{\prime} \mid\left(s^{\prime} \mathrm{I} A\right) \neq \phi\right\}\right)-Q(\{s \subseteq B \mid(s \text { I } A) \neq \phi\}),
$$

where $B=(p, W)$ and $B^{\prime}=B\left(p^{\prime}, W^{\prime}\right)$.

To see the intuition of WASRP, consider two budget sets $B, B^{\prime}$. Let $A$ denote any collection of consumption bundles available under both budget sets. Suppose the probability of choosing a set that contains at least one element of $A$ falls when the budget set changes from $B^{\prime}$ to $B$. Then, in effect, the consumer rejects all bundles in A more frequently than earlier. Such greater rejection cannot be in favor of bundles in $\left[\left(B \mathrm{I} B^{\prime}\right) \backslash A\right]$ : these were available earlier as well. Hence, this must be in favor of bundles that were earlier unavailable. The magnitude of the fall should not, therefore, exceed the probability of choosing a set, under $B$, that only contains bundles previously unavailable.

Notice now that (5.1) is equivalent to:

$$
Q\left(\left\{s \subseteq B \mid\left(s \text { I } B^{\prime}\right) \neq \phi=(s \text { I } A)\right\}\right) \leq Q^{\prime}\left(\left\{s^{\prime} \subseteq B^{\prime} \mid\left(s^{\prime} \mathrm{I} A\right)=\phi\right\}\right) .
$$


Thus, WASRP also requires the probability of rejecting all bundles in $A$ under $B^{\prime}$ to be no less than that of rejecting them, under $B$, in favor of at least one bundle also available under $B^{\prime}$.

Rationality postulates analogous to our WASRP for SDCs have been developed for SDFs, DDCs and DDFs. We now clarify their connections with our rationality postulate for SDCs.

\section{Definition 5.2.}

(i) An SDF, $D$, satisfies the weak axiom of stochastic revealed preference (WASRP) iff, for all $(p, W),\left(p^{\prime}, W^{\prime}\right) \in Z$, and for every $A \subseteq\left(B I B^{\prime}\right)$,

$$
q\left(B \backslash B^{\prime}\right) \geq q^{\prime}(A)-q(A) .
$$

(ii) A DDC, $c$, satisfies the weak axiom of revealed preference (WARP) iff, for all $(p, W),\left(p^{\prime}, W^{\prime}\right) \in Z$, if $\left[c(p, W) \mathrm{I} B^{\prime}\right] \neq \phi$, then:

$$
\left[c\left(p^{\prime}, W^{\prime}\right) \mathrm{I} \quad B\right]=\phi \text { when }\left[c\left(p^{\prime}, W^{\prime}\right) \mathrm{I} \quad B\right] \neq\left[c(p, W) \mathrm{I} \quad B^{\prime}\right] \text {. }
$$

(iii) A DDF, $d$, satisfies the weak axiom of revealed preference (WARP) iff, for all $(p, W),\left(p^{\prime}, W^{\prime}\right) \in Z$, if $d(p, W) \in B^{\prime}$, then:

$$
d\left(p^{\prime}, W^{\prime}\right) \in\left[\left(B^{\prime} \backslash B\right) Y\{d(p, W)\}\right] .
$$

Bandyopadhyay et al. (1999) introduced WASRP for SDFs (Definition 5.2(i)). This requires the probability, under $B^{\prime}$, of the chosen consumption bundle lying in some subset of ( $B$ I $\left.B^{\prime}\right)$, not to exceed the probability, under $B$, of the chosen bundle either lying in that subset or being unavailable under $B^{\prime}$. WARP for DDCs, as specified in Definition 5.2(ii), is equivalent to both Richter's (1966) ‘weak congruence axiom’ and Sen’s (1971) specification of WARP (except that Sen considers general choice problems, not the specific problem of choice by a competitive consumer). This requires the following. If a consumption bundle is rejected in one situation, it cannot be chosen in another, so long as some bundle chosen in the first situation is also available in the second. This condition is a straightforward extension of the classical WARP for DDFs. Observed market purchases of a consumer who chooses according to a tight DDC satisfying WARP, and (deterministically) purchases a consumption bundle from her choice set, must satisfy a weaker version of Varian's (1982) GARP, which is required to hold only over all pairs of price-wealth situations. Our statement of WARP for DDFs (Definition 5.2(iii)) is equivalent to the original weak axiom of revealed preference due to Samuelson (1938). This requires, when the (unique) consumption bundle chosen under $B$ is also available under $B^{\prime}$, the (unique) consumption bundle chosen under $B^{\prime}$ must either be identical to that chosen under $B$, or else be unavailable under $B$. 
Remark 5.3. If a degenerate SDC, $C$, satisfies WASRP, then the DDC induced by $C$ must satisfy WARP. If a singular SDC, $C$, satisfies WASRP, then the SDF induced by $C$ must satisfy WASRP. If a singular and degenerate SDC, $C$, satisfies WASRP, the DDF induced by $C$ must satisfy WARP. ${ }^{9}$

The SDF-based and DDC-based frameworks, via their corresponding versions of choice consistency in terms of the weak axiom, extend the traditional DDF-based framework along different dimensions, in that neither subsumes the other. This is intuitively self-evident from an a priori perspective, but not immediately so from an ex post explanatory perspective, since observed market purchases cannot directly reveal multi-element choice, but can only be interpreted in such terms in order to ascribe consistency. First note that, if the consumer's observed market purchases are deterministic and involve wealth exhaustion, yet violate WARP for DDFs, they cannot be represented by any tight (degenerate) SDF satisfying WASRP. However, following the approach pioneered by Afriat (1967) and Varian (1982), such purchases may still be open to rationalization in terms of a tight DDC satisfying WARP. Intuitively, the presumption then is, when the consumer's choice set has several elements, she picks a bundle out of this multi-element set for actual purchase according to some unspecified behavioral rule. So long as the theorist can, ex post, construct a tight DDC satisfying WARP and a behavioral rule that, together, imply observed demand behavior, she can then claim that what appears to be a tight DDF violating classical WARP (or a tight (degenerate) SDF violating WASRP) is actually a tight DDC satisfying WARP together with this behavioral rule. But can one then, in the same spirit, also rationalize the consumer's market purchases in terms of a tight DDC satisfying WARP, whenever she is observed to purchase according to a (non-degenerate) tight SDF satisfying WASRP? The question thus naturally arises whether there exist classes of market demand behavior that can be generated by some tight SDF satisfying WASRP, but which cannot possibly be generated by any tight DDC satisfying WARP, regardless of the behavioral rule linking actual market choices to choice sets with multiple elements. There is a second issue at stake as well. Our SDC-based approach, encompassing all the other approaches as alternative special cases, can evidently accommodate all forms of choice, as well as observed market purchase, behavior that the other three can. Thus, the advantage of theoretical unification that our approach offers is evident. Does it additionally offer the advantage of expansion in terms of empirical applicability in a market purchase context? In other words, are there classes of market behavior that cannot be generated by any tight DDF, DDC or SDF satisfying the relevant version of the weak axiom, but which are consistent with choice behavior according to some tight SDC satisfying WASRP, along with particular behavioral rules for determining actual market purchase out of multi-element choice sets?

We now proceed to address these issues. We first provide two examples to justify our claim that the SDF based approach can accommodate some classes of observed market behavior that the

\footnotetext{
${ }^{9}$ The last claim is easy to check. Lemmas N.1(i) and N.2(i) in the Appendix provide proofs of the first two.
} 
DDC based one cannot, and vice versa. Combining these two examples, our third example establishes that the SDC based approach can indeed accommodate forms of observed market behavior that the other three cannot. Thus, our behavioral restriction for SDCs, i.e. WASRP, offers the dual advantages of unification and expansion beyond all three existing versions from both the intuitive, a priori, and the interpretative, a posteriori, observed market behavior, perspectives.

We consider a three-commodity world, where the consumer never purchases the third commodity. ${ }^{10}$ In Example 5.4 below, we provide a complete specification of market purchases for all price-wealth situations in this world, which could not have been generated by either a tight DDF or a tight DDC that satisfies the relevant version of WARP. This specification nevertheless is compatible with the consumer choosing according to either a tight (non-degenerate) SDF or a tight (singular and non-degenerate) SDC satisfying WASRP. Intuitively, the market behavior specified is what would come about if the consumer were to randomize among alternative lexicographic preference orderings. In Example 5.5, we consider market behavior that can neither be generated by a tight SDF satisfying WASRP, nor by a tight DDF satisfying WARP. We show that such market behavior would nevertheless come about if the consumer was choosing either according to a tight DDC satisfying WARP, or a (degenerate and non-singular) SDC satisfying WASRP, and was purchasing a bundle out of her multi-element choice set according to a particular behavioral rule. Intuitively, the demand behavior specified could occur due to preferences that ascribe a constant marginal rate of substitution between commodities 1 and 2. Lastly, we provide an example of observed market behavior that could not have been generated by a tight DDF satisfying WARP, nor by a tight DDC satisfying WARP, nor indeed, by a tight SDF satisfying WASRP. However, such market behavior is consistent with the consumer choosing according to a tight (non-singular and non-degenerate) SDC satisfying WASRP, and purchasing a bundle out of her multi-element choice set according to a particular behavioral rule. The demand behavior specified might come about when the consumer is randomizing among the alternative preference relations that rationalize the first two examples.

\section{Example 5.4.}

Given any $(p, W) \in Z$, suppose the consumer is observed to purchase the consumption bundle $\bar{x}=\left(\frac{W}{p_{1}}, 0,0\right)$ with probability $\mu \in(0,1)$, and the bundle $\underline{x}=\left(0, \frac{W}{p_{2}}, 0\right)$ with probability $(1-\mu)$. Clearly, WARP in its classical, DDF form, becomes inapplicable. Then one can model this demand behavior in terms of an SDF such that, for every $(p, W) \in Z, q(\{\bar{x}(p, W)\})=\mu$, $q(\{\underline{x}(p, W)\})=1-\mu$. One may also model such behavior in terms of a singular and non-degenerate SDC, such that, for all $(p, W) \in Z, Q(\{\{\bar{x}(p, W)\}\})=\mu, Q(\{\{\underline{x}(p, W)\}\})=1-\mu$. It can be checked

${ }^{10}$ Our examples can be easily extended to any world with more than three commodities. 
that, in either case, tightness and WASRP must both be satisfied. Suppose, instead, one tries to fit such behavior into a deterministic choice framework by assuming: (i) the consumer is actually choosing, under $(p, W)$, some subset $A$ of the budget set $B(p, W)$, and (ii) purchasing some element out of $A$ according to some probabilistic behavioral rule. Then, since $\mu \in(0,1)$, it must be that $A \supseteq\{\bar{x}(p, W), \underline{x}(p, W)\}$. It can be checked that, given tightness, every DDC that can be thus constructed to rationalize observed purchases must necessarily violate the relevant version of WARP.

\section{Example 5.5.}

Suppose now that, for every $(p, W) \in Z$, the consumer's observed market purchases are as follows: (i) if $\frac{p_{2}}{p_{1}}>1$ or $\left[\frac{p_{2}}{p_{1}}=1\right.$ and $\left.p_{3} \leq 1\right]$, the purchased bundle is $\left(\frac{W}{p_{1}}, 0,0\right)$; and (ii) if $\frac{p_{2}}{p_{1}}<1$ or $\left[\frac{p_{2}}{p_{1}}=1\right.$ and $\left.p_{3}>1\right]$, the purchased bundle is $\left(0, \frac{W}{p_{2}}, 0\right)$. It can be checked that the consumer's market behavior satisfies tightness, but violates Samuelson's WARP. Since the consumer exhibits no probabilistic element in her purchases, if her market purchases are modeled by an SDF, such an SDF must be degenerate. Since the DDF specification violates WARP, the degenerate SDF specification must violate WASRP. Suppose now that one ascribed the following DDC to the consumer: (i) if $\frac{p_{2}}{p_{1}}>1$, the choice set is $\left\{\left(\frac{W}{p_{1}}, 0,0\right)\right\}$; (ii) if $\frac{p_{2}}{p_{1}}<1$, the choice set is $\left\{\left(0, \frac{W}{p_{2}}, 0\right)\right\}$; (iii) if $\frac{p_{2}}{p_{1}}=1$, the choice set is $\left\{x \in \mathfrak{R}_{+}^{3} \mid p x=W, x_{3}=0\right\}$. Suppose further that one ascribed the obvious behavioral rule, consistent with observed purchases, for deciding which bundle to buy from the choice set when $\frac{p_{2}}{p_{1}}=1$. It can be checked that such a DDC, while consistent with market behavior, also satisfies both WARP and tightness. One could analogously define a degenerate and non-singular SDC that satisfies both WASRP and tightness, while rationalizing market purchases.

\section{Example 5.6.}

Suppose now that, for every $(p, W) \in Z$, observed market purchases are as follows: for $\mu, \eta \in(0,1)$, (i) if $\frac{p_{2}}{p_{1}}>1$, or $\left[\frac{p_{2}}{p_{1}}=1\right.$ and $\left.p_{3} \leq 1\right]$, the purchased bundle is $\left(\frac{W}{p_{1}}, 0,0\right)$ with probability $\mu \eta+(1-\eta)$ and $\left(0, \frac{W}{p_{2}}, 0\right)$ with probability $(1-\mu) \eta$; (ii) if $\frac{p_{2}}{p_{1}}<1$, or $\left[\frac{p_{2}}{p_{1}}=1\right.$ and 
$p_{3}>1$ ], the purchased bundle is $\left(\frac{W}{p_{1}}, 0,0\right)$ with probability $\mu \eta$ and $\left(0, \frac{W}{p_{2}}, 0\right)$ with probability $(1-\mu) \eta+(1-\eta)$. In light of Examples 5.4 and 5.5, it can be seen that, assuming tightness, neither a DDF satisfying WARP, nor an SDF satisfying WASRP, nor, indeed, a DDC satisfying WARP could have generated the market demand behavior specified. Such demand behavior could however come about if the consumer were to randomize between the two SDCs that rationalize demand behavior in the earlier two examples, choosing according to the (singular) SDC specified in Example 5.4 with probability $\eta$. Since both these SDCs satisfy tightness and WASRP, such randomization in turn must generate a (non-singular and non-degenerate) SDC that satisfies both tightness and WASRP.

\section{Results}

We are now ready to present our substantive results.

Proposition 6.1. (General Substitution Theorem) A tight SDC, C, satisfies WASRP if and only if it also satisfies SS.

Proof: See the Appendix.

The General Substitution Theorem (Proposition 6.1) is our central result. Under the assumption of tightness, it completely specifies the restrictions on demand behavior imposed by WASRP when applied to SDCs. It provides a central unifying result, in that a number of key results in the theory of consumer's behavior can be shown to stem from this result.

First notice that Proposition 6.1 yields three basic results in demand theory, non-positivity of the own substitution effect, the demand theorem, and homogeneity of degree zero, for SDCs.

Corollary 6.2. Suppose a tight SDC, C, satisfies WASRP. Then:

(i) C must satisfy NPS;

(ii) every normal good must also be regular, and

(iii) C must be homogeneous of degree zero in prices and wealth.

Proof: See the Appendix.

Counterparts of these results for DDCs can also be derived from the General Substitution Theorem, as we now formally note. Since the theory of demand behavior with DDCs appears to have escaped attention in the literature, this case is of independent interest as well. Recall Notation 4.6. 
Corollary 6.3. Let $c$ be a tight DDC.

(i) (Deterministic Substitution Theorem) $c$ satisfies WARP iff, for all $(p, W),\left(p^{\prime}, W^{\prime}\right) \in Z$ such that $p^{\prime} . x \leq W^{\prime}$ for some $x \in c(p, W)$ :

$$
c\left(p^{\prime}, W^{\prime}\right) \subseteq\left[G^{\prime} \mathrm{Y} I\right] \text {, and }\left[c\left(p^{\prime}, W^{\prime}\right) \subseteq G^{\prime} \text { if }\left[c\left(p^{\prime}, W^{\prime}\right) \mathrm{I} I\right] \neq[c(p, W) \mathrm{I} I]\right] .
$$

(ii) Let $c$ satisfy WARP. Then, for every $i \in M$, for all $(p, W),\left(p^{\prime}, W^{\prime}\right) \in Z$ such that $\left[\left[p, p^{\prime}\right.\right.$ are $i$-variant with $\left.p_{i}^{\prime}<p_{i}\right]$, and $\left[p^{\prime} \cdot x^{*}=W^{\prime}\right.$ for some $\left.\left.x^{*} \in c(p, W)\right]\right]$, and for all $x^{\prime} \in c\left(p^{\prime}, W^{\prime}\right)$,

$$
\left[x_{i}^{*} \leq x_{i}^{\prime}\right] \text {; the inequality holding strictly when }\left[c\left(p^{\prime}, W^{\prime}\right) \mathrm{I} I\right] \neq[c(p, W) \mathrm{I} I] \text {. }
$$

(iii) Let $c$ satisfies WARP, and suppose $i \in M$ is normal according to the degenerate SDC induced by $c$. Then, for all $p, p^{\prime} \in \mathfrak{R}_{++}^{m}$ such that $\left[p, p^{\prime}\right.$ are $i$-variant with $\left.p_{i}^{\prime}<p_{i}\right]$, for every $W \in \mathfrak{R}_{+}$, and for all $x^{\prime} \in c\left(p^{\prime}, W\right)$,

$$
\left[\sup \left\{x_{i} \mid x \in c(p, W)\right\} \leq x_{i}^{\prime}\right] .
$$

(iv) If c satisfies WARP, it must be homogeneous of degree 0 in prices and wealth.

Proof: See the Appendix.

Notice that, given tightness, $\left[c\left(p^{\prime}, W^{\prime}\right) \mathrm{I} I\right] \neq[c(p, W) \mathrm{I} I]$ when $p^{\prime} . x<W^{\prime}$ for some $x \in c(p, W)$. Then Corollary 6.3(i) implies that $c\left(p^{\prime}, W^{\prime}\right) \subseteq G^{\prime}$ if, and only if, the DDC satisfies WARP. Notice also the forms the familiar non-positivity property of the own substitution effect and the demand theorem acquire in the context of deterministic demand correspondences (Corollary 6.3(ii) and Corollary 6.3(iii)). Suppose the price of commodity $i$ falls, all other prices remaining constant. Suppose one took any arbitrary consumption bundle that was initially chosen as the reference bundle, and reduced the consumer's wealth, so that this reference bundle cost exactly the consumer's wealth in the new situation. Then, given WARP, no consumption bundle chosen in the new situation can contain less of the $i$-th commodity than the amount contained in the reference bundle. If $\left[c\left(p^{\prime}, W^{\prime}\right) \mathrm{I} I\right] \neq[c(p, W) \mathrm{I} I]$, then every bundle chosen in the new situation must contain strictly more. An analogous weak set-dominance condition characterizes the demand theorem: given wealth and all other prices, no consumption bundle chosen subsequent to a fall in the price of a normal commodity can contain less of that commodity, compared to the amount contained in any consumption bundle chosen initially.

The central result of Bandyopadhyay, Dasgupta and Pattanaik (2004) also follows as a special case from Proposition 6.1, as we now specify. Recall Notation 4.6. 
Corollary 6.4. Let $D$ be a tight SDF.

(i) (Stochastic Substitution Theorem) D satisfies WASRP iff it also satisfies the following: for all $(p, W),\left(p^{\prime}, W^{\prime}\right) \in Z$, and for every $A \subseteq I$,

$$
\left[q^{\prime}\left(G^{\prime}\right)+q^{\prime}(A) \geq q(H)+q(A)\right] .
$$

(ii) If D satisfies WASRP, it must be homogeneous of degree zero in prices and wealth.

Proof: See the Appendix.

Bandyopadhyay, Dasgupta and Pattanaik (2004) show that the Stochastic Substitution Theorem (Corollary 6.4(i)) generates: (a) non-positivity of the own substitution effect and the demand theorem for SDFs, (b) equivalence of WARP with Samuelson's Inequality for DDFs (which we have termed Samuelson's Substitution Theorem) and (c) non-positivity of the own substitution effect and the demand theorem for DDFs. Obviously, Corollary 6.4(ii) yields H(0) for DDFs as a special case. It follows that these key results in the theory of consumer's behavior all follow as special cases of our General Substitution Theorem (Proposition 6.1). Notice that the results for DDFs, i.e. (b) and (c) above, as well as $\mathrm{H}(0)$, can also be alternatively generated from our Corollary 6.3 as the special case where the DDC is additionally constrained to be singular. Furthermore, (a) and (c) above, and Corollary 6.3 ((ii) and (iii)) can all be generated as special cases of Corollary 6.2. H(0) in every case can be also be generated from Corollary 6.2(iii).

The interconnections between these various results are summarized in Figure 2. As is evident from Figure 2, our General Substitution Theorem (Proposition 6.1) provides the core unifying result, which yields all the other results as special cases.

\section{Insert Figure 2}

Notice that, to interpret our results in terms of observed market behavior of the consumer, we do not need any specific behavioral rule for linking a realized multi-element choice set and the consumption bundle that the consumer actually buys, beyond the obvious, minimal one that the purchased bundle be a member of the realized choice set. The relationships we have specified hold for all members of the realized choice set, including, obviously, the one actually purchased. Thus, the set-dominance criteria we utilize ensure empirical applicability of our results directly in terms of observed market purchases, irrespective of the behavioral rule followed by the consumer in translating multi-element choice into actual market purchase. Conversely, so long as the consumer's observed market purchases can be rationalized in terms of some tight SDC that satisfies SS, one cannot falsify 
the hypothesis that she is indeed choosing according to that tight SDC, which must satisfy WASRP. ${ }^{11}$ One may then make the standard inductive move in using our results to predict the consumer's market purchases in as-yet-unobserved situations. As pointed out earlier in Section 5, this may be possible even when her observed market purchases violate the weak axiom in its three other, stronger, versions, thereby making theories based on such versions useless for generating testable predictions.

\section{Conclusion}

In this paper, we have integrated and expanded the WARP-based theory of consumer's behavior to simultaneously cover both probabilistic choice and choice of multiple consumption bundles. We have offered a consistency postulate for demand behavior when such behavior is represented in terms of a stochastic demand correspondence. We have shown show that, when the consumer spends her entire wealth with probability one, our rationality postulate is equivalent to a condition we have termed stochastic substitutability. This equivalence generates: (i) Samuelson's Substitution Theorem, (ii) the central result in Bandyopadhyay, Dasgupta and Pattanaik (2004) and (iii) a version pertinent to deterministic demand correspondences, which independently yields Samuelson's Substitution Theorem, as alternative special cases. Relevant versions of the non-positivity property of the own substitution effect, the demand theorem, and the property of homogeneity of degree zero in all prices and wealth, all fall out as corollaries in every case. Thus, we have provided a core unifying result, which subsumes and expands available results. This result may perhaps be seen as providing a logical closure to the WARP-based analysis of demand behavior initiated by Samuelson (1938).

Extension of our analysis to the issue of rationalizability in terms of complete but not necessarily transitive stochastic preference relations would appear to be a natural next step, as would the construction of a stochastic counterpart of the deterministic Strong Axiom of Revealed Preference. Application of our rationality postulate to the problem of aggregating demand correspondences, along the lines of Bandyopadhyay, Dasgupta and Pattanaik (2002), may constitute another useful line of investigation. Contexts where the budget set is subject to random shocks, and issues regarding the relationship between our rationality postulate and weaker notions in general choice contexts, along the lines of Dasgupta and Pattanaik (2007), can also be explored. We leave these issues for the future.

11 It can be shown that, if observed market purchases are consistent with an SDC which, given any ordered pair $\left\langle(p, W),\left(p^{\prime}, W^{\prime}\right)\right\rangle \in Z^{2}$, satisfies (4.3) for every $A \in\{\phi, I\}$, then they must also be consistent with an SDC satisfying SS, and hence (by Proposition 6.1), WASRP. Thus, rationalization of observed market purchases in terms of an SDC satisfying WASRP requires one only to construct an SDC which, besides being consistent with such purchases, also satisfies (4.3) for every ordered pair $\left\langle(p, W),\left(p^{\prime}, W^{\prime}\right)\right\rangle \in Z^{2}$ and for every $A \in\{\phi, I\}$. It can be seen that this condition constitutes the natural stochastic counterpart of Varian's (1982) (deterministic) GARP, when GARP is weakened to apply only to all pairs of price-wealth situations. 


\section{Appendix}

Throughout the proofs, we shall use the notation presented in Notation 4.6.

\section{Proof of Proposition 6.1.}

Let the SDC, $C$, be tight. Then, for any $A \subseteq I$ :

$$
\begin{gathered}
Q(r(G))+Q\left(\left\{s \subseteq B \mid\left(s \text { I }\left(H^{\prime} \mathrm{Y} A\right)\right) \neq \phi\right\}\right)+Q(\{s \subseteq B \mid \phi \neq(s \text { I I }) \subseteq(I \backslash A)\})+ \\
Q(\{s \subseteq B \mid(s \text { I } I)=\phi,(s \text { I } H) \neq \phi\})=1 ; \\
Q^{\prime}\left(r\left(G^{\prime}\right)\right)+Q^{\prime}\left(\left\{s^{\prime} \subseteq B^{\prime} \mid\left(s^{\prime} \mathrm{I}\left(H^{\prime} \mathrm{Y} A\right)\right) \neq \phi\right\}\right)+ \\
Q^{\prime}\left(\left\{s^{\prime} \subseteq\left(G^{\prime} \mathrm{Y} I\right) \mid \phi \neq\left(s^{\prime} \mathrm{I} I\right) \subseteq(I \backslash A)\right\}\right)=1 .
\end{gathered}
$$

First suppose $C$ satisfies WASRP. Since $\left[H^{\prime} \mathrm{Y} A\right] \in r\left(B \mathrm{I} B^{\prime}\right)$, by WASRP, we have:

$$
Q(r(G))+Q\left(\left\{s \subseteq B \mid\left(s \text { I }\left(H^{\prime} \mathrm{Y} A\right)\right) \neq \phi\right\}\right) \geq Q^{\prime}\left(\left\{s^{\prime} \subseteq B^{\prime} \mid\left(s \text { I }\left(H^{\prime} \mathrm{Y} A\right)\right) \neq \phi\right\}\right) .
$$

Together, (N1)-(N3) imply (4.3) for any $[I \backslash A] \subseteq I$.

Now suppose $C$ satisfies SS. Consider any $A \in r\left(B I B^{\prime}\right)$; let $A_{0}=A$ I $I$. By tightness,

$$
Q\left(\left\{s \subseteq B \mid\left(s \mathrm{I}\left(H^{\prime} \mathrm{Y} A_{0}\right)\right) \neq \phi\right\}\right)=Q\left(\left\{s \subseteq B \mid\left(s \mathrm{I} A_{0}\right) \neq \phi\right\}\right) .
$$

Noting (N1), (N2) and (N4), by SS (4.3), we get:

$$
Q(r(G))+Q\left(\left\{s \subseteq B \mid\left(s \text { I } A_{0}\right) \neq \phi\right\}\right) \geq Q^{\prime}\left(\left\{s^{\prime} \subseteq B^{\prime} \mid\left(s \text { I }\left(H^{\prime} \mathrm{Y} A_{0}\right)\right) \neq \phi\right\}\right) .
$$

Since, by construction, $A_{0} \subseteq A$, and since $C$ is tight, we get, respectively,

$$
\begin{aligned}
& Q(\{s \subseteq B \mid(s \mathrm{I} A) \neq \phi\}) \geq Q\left(\left\{s \subseteq B \mid\left(s \mathrm{I} A_{0}\right) \neq \phi\right\}\right), \\
& Q^{\prime}\left(\left\{s^{\prime} \subseteq B^{\prime} \mid\left(s^{\prime} \mathrm{I}\left(H^{\prime} \mathrm{Y} A_{0}\right)\right) \neq \phi\right\}\right) \geq Q^{\prime}\left(\left\{s^{\prime} \subseteq B^{\prime} \mid\left(s^{\prime} \mathrm{I} A\right) \neq \phi\right\}\right) .
\end{aligned}
$$

Together, (N5)-(N7) imply (5.1).

\section{Proof of Corollary 6.2.}

(i) Consider any $(p, W),\left(p^{\prime}, W^{\prime}\right) \in Z$ such that, for some $i \in M,\left[p, p^{\prime}\right.$ are $i$-variant with $p_{i}^{\prime}<p_{i}$, and $\alpha \equiv\left(\frac{W-W^{\prime}}{p_{i}-p_{i}^{\prime}}\right) \in\left[0, \frac{W}{p_{i}}\right]$. Let the tight SDC, C, satisfy WASRP. Then, by Proposition 6.1, C satisfies SS. It can be checked that, (i) for all $x^{\prime} \in G^{\prime}, x_{i}^{\prime}>\alpha$, (ii) for all $x \in H$, $x_{i}>\alpha$, (iii) for all $x \in I, x_{i}=\alpha$, (iv) for all $x^{\prime} \in H^{\prime}, x_{i}^{\prime}<\alpha$, and (v) for all $x \in G, x_{i}<\alpha$. Noting $C$ is tight, (4.3) yields (4.1) when $A=\phi$, and (4.2) when $A=I$.

(ii) Part (ii) follows from Corollary 6.2(i), Definition 4.4 and Definition 4.5.

(iii) The proof is obvious and therefore omitted.

We shall prove Corollary 6.3 via the following Lemma. 


\section{Lemma N.1.}

(i) A degenerate SDC, $C$, satisfies WASRP iff the DDC induced by $C$ satisfies WARP.

(ii) A tight and degenerate SDC, $C$, satisfies SS iff the DDC induced by $C$ satisfies the following: for all $(p, W),\left(p^{\prime}, W^{\prime}\right) \in Z$, such that $p^{\prime} . x \leq W^{\prime}$ for some $x \in c(p, W)$, (6.1) holds.

Proof of Lemma N.1. Let $C$ be a degenerate SDC, and let $c$ be the DDC induced by $C$. Then the degenerate SDC induced by $c$ must be $C$ itself. Consider any $(p, W),\left(p^{\prime}, W^{\prime}\right) \in Z$. Then, for some $a \in r(B), Q(\{a\})=1$, and for some $a^{\prime} \in r\left(B^{\prime}\right), Q^{\prime}\left(\left\{a^{\prime}\right\}\right)=1$; also, $c(p, W)=a$ and $c\left(p^{\prime}, W^{\prime}\right)=a^{\prime}$. (i) First let $C$ satisfy WASRP, and suppose $\left[\begin{array}{ll}a \mathrm{I} & B^{\prime}\end{array}\right] \neq \phi, \phi \neq\left[\begin{array}{ll}a^{\prime} \mathrm{I} & B\end{array}\right] \neq\left[\begin{array}{ll}a \mathrm{I} & B^{\prime}\end{array}\right]$. Without loss of generality, suppose $\tilde{a} \equiv\left[\left[\begin{array}{ll}a^{\prime} \mathrm{I} & B\end{array}\right] \backslash a\right] \neq \phi$. Then $Q\left(r\left(B \backslash B^{\prime}\right)\right)=Q(\{s \subseteq B \mid(s \mathrm{I} \tilde{a}) \neq \phi\})=0$, but $Q^{\prime}\left(\left\{s^{\prime} \subseteq B^{\prime} \mid\left(s^{\prime} \mathrm{I} \tilde{a}\right) \neq \phi\right\}\right)=1$, a violation of WASRP.

Now let $c$ satisfy WARP. Suppose $\left[a \mathrm{I} B^{\prime}\right] \neq \phi$. Then, by WARP: (i) for $A \in r\left(B \mathrm{I} B^{\prime}\right)$ such that $[a \mathrm{I} A]=\phi$, the RHS of (5.1) is 0 , and (ii) for $A \in r\left(B \mathrm{I} B^{\prime}\right)$ such that $\left[\begin{array}{ll}a \mathrm{I} & A\end{array}\right] \neq \phi$, the LHS of (5.1) is 1 . Thus, in all cases, (5.1) must hold. If $\left[\begin{array}{ll}a & B^{\prime}\end{array}\right]=\phi$, the claim holds trivially.

(ii) Let $C$ be tight. First let $C$ satisfy SS. Suppose $p^{\prime} . x \leq W^{\prime}$ for some $x \in a$. By tightness, $\phi \neq\left[a \mathrm{I} \quad B^{\prime}\right] \subseteq(H \mathrm{Y} I)$. Then, by tightness and SS (putting $\left.A=I\right)$, we have $a^{\prime} \subseteq\left[G^{\prime} \mathrm{Y} I\right]$. Now suppose $\left(a \mathrm{I} B^{\prime}\right) \neq\left(a^{\prime} \mathrm{I} B\right) \neq \phi$. Then either $[a \mathrm{I} H] \neq \phi$, or $\left[\begin{array}{ll}a \mathrm{I} & I\end{array}\right] \neq\left[\begin{array}{ll}a^{\prime} \mathrm{I} & I\end{array}\right]$, or both. If

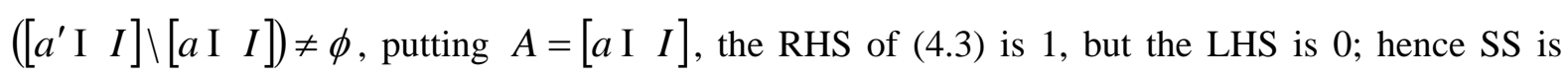
violated. It can be checked that SS is analogously violated in the other cases as well.

Now let $c$ satisfy (6.1) when $p^{\prime} . x \leq W^{\prime}$ for some $x \in c(p, W)$. First suppose $p^{\prime} . x<W^{\prime}$ for some $x \in c(p, W)$. Then, by (6.1), $Q^{\prime}\left(r\left(G^{\prime}\right)\right)=1:$ (4.3) must hold for all $A \subseteq I$. Now suppose $p^{\prime} . x \leq W^{\prime}$ for some $x \in c(p, W)$, and $p^{\prime} . x \geq W^{\prime}$ for every $x \in c(p, W)$. First suppose $\left[c\left(p^{\prime}, W^{\prime}\right) \mathrm{I} I\right] \neq[c(p, W) \mathrm{I} I]$. Then, by (6.1), $Q^{\prime}\left(r\left(G^{\prime}\right)\right)=1:$ (4.3) must hold for all $A \subseteq I$. Now let $\left[c\left(p^{\prime}, W^{\prime}\right) \mathrm{I} I\right]=[c(p, W) \mathrm{I} I]$. Consider any $A \subseteq I$. If $[c(p, W) \mathrm{I} I] \subseteq A$, by (6.1), the LHS of (4.3) is 1. If $[c(p, W) I I] \not \subset A$, the RHS of (4.3) is 0 . Thus, in either case, (4.3) holds. Lastly, if $p^{\prime} . x>W^{\prime}$ for every $x \in c(p, W)$, then the RHS of (4.3) must be 0. Hence, (4.3) must hold.

\section{Proof of Corollary 6.3.}

Proposition 6.1 and Lemma N.1 yield part (i). Part (i) yields part (ii), part (iii) follows from part (ii) and Definition 4.4; part (iv) follows from Lemma N.1(i), Corollary 6.2(iii) and Definition 4.2. $\quad \diamond$ 
We shall prove Corollary 6.4 via the following Lemma.

\section{Lemma N.2.}

(i) A singular SDC, $C$, satisfies WASRP iff the SDF induced by $C$ satisfies WASRP.

(ii) A tight and singular SDC, $C$, satisfies SS iff the SDF induced by $C$ satisfies (6.2).

Proof of Lemma N.2. Let $C$ be a singular SDC, and let $D$ be the SDF induced by $C$. Then the singular SDC induced by $D$ must be $C$. Consider any $(p, W),\left(p^{\prime}, W^{\prime}\right) \in Z$.

(i) First let $C$ satisfy WASRP. Consider any $A \in r\left[B I B^{\prime}\right]$. Since $C$ is singular, we get:

$$
\begin{aligned}
& Q(\{s \subseteq B \mid(s \text { I } A) \neq \phi\})+Q\left(r\left(B \backslash B^{\prime}\right)\right)=q(A)+q\left(B \backslash B^{\prime}\right), \\
& Q^{\prime}\left(\left\{s^{\prime} \subseteq B^{\prime} \mid\left(s^{\prime} \mathrm{I} A\right) \neq \phi\right\}\right)=q^{\prime}(A), \\
& Q\left(r\left(B \backslash B^{\prime}\right)\right)=q\left(B \backslash B^{\prime}\right) .
\end{aligned}
$$

Noting that $\left(q^{\prime}(\phi)=0\right)$, part (i) of Lemma N.2 follows immediately from (N8)-(N10)

(ii) Let $C$ be tight. Suppose $C$ satisfies SS. Consider any $A \subseteq I$. Since $C$ is singular:

$$
\begin{aligned}
& Q^{\prime}\left(\left\{s^{\prime} \subseteq\left(G^{\prime} \mathrm{Y} I\right) \mid \phi \neq\left(s^{\prime} \mathrm{I} I\right) \subseteq A\right\}\right)=q^{\prime}(A), \\
& Q(\{s \subseteq B \mid \phi \neq(s \text { I } I) \subseteq A\})=q(A), \\
& Q^{\prime}\left(r\left(G^{\prime}\right)\right)=q^{\prime}\left(G^{\prime}\right), \\
& Q(\{s \subseteq B \mid(s \text { I I })=\phi,(s \text { I } H) \neq \phi\})=q(H) .
\end{aligned}
$$

Part (ii) of Lemma N.2 follows directly from (N11)-(N14).

\section{Proof of Corollary 6.4.}

Proposition 6.1 and Lemma N.2 together yield part (i), while part (ii) follows from Corollary 6.2(iii), Lemma N.2(i) and Definition 4.2. 


\section{References}

Afriat, S.N. (1967): “The construction of a utility function from expenditure data”, International Economic Review 8: 67-77.

Arrow, K. (1959): “Rational choice functions and orderings”, Economica (N.S.) 26: 121-127.

Bandyopadhyay, T., I. Dasgupta and P.K. Pattanaik (2004): “A general revealed preference theorem for stochastic demand behavior”, Economic Theory 23: 589-599.

Bandyopadhyay, T., I. Dasgupta and P.K. Pattanaik (2002): “Demand aggregation and the weak axiom of stochastic revealed preference”, Journal of Economic Theory 107: 483-489.

Bandyopadhyay, T., I. Dasgupta and P.K. Pattanaik (1999): "Stochastic revealed preference and the theory of demand”, Journal of Economic Theory 84: 95-110.

Barbera, S. and P. K. Pattanaik (1986): "Falmagne and the rationalizability of stochastic choices in terms of random orderings”, Econometrica 54: 707-715.

Block, H. D. and J. Marschak (1960): “Random orderings and stochastic theories of response”, in

I. Olkin, S. Ghurye, W. Hoeffding, W. Madow and H. Mann (eds) Contributions to Probability and Statistics (Stanford: Stanford University Press).

Cohen, M. A. (1980): “Random utility systems: the infinite case”, Journal of Mathematical Psychology 22: 1-23.

Corbin, R. and A. A. J. Marley (1974): "Random utility models with equality: an apparent, but not actual, generalization of random utility models”, Journal of Mathematical Psychology 11: 274-293.

Dasgupta, I. (2005): “Consistent firm choice and the theory of supply”, Economic Theory 26: 167175.

Dasgupta, I. and P.K. Pattanaik (2007): “'Regular' choice and the weak axiom of stochastic revealed preference”, Economic Theory 31: 35-50.

Falmagne, J. C. (1978): “A representation theorem for finite random scale systems”, Journal of Mathematical Psychology 18: 52-72.

Fishburn, P. (1978): “Choice probabilities and choice functions”, Journal of Mathematical Psychology 18: 205-219.

Fishburn, P. (1977): “Models of individual preference and choice”, Synthese 36: 287-314.

Fishburn, P. (1973): "Binary choice probabilities: on the varieties of stochastic transitivity”, Journal of Mathematical Psychology 10: 327-352.

Halldin, C. (1974): “The choice axiom, revealed preference and the theory of demand”, Theory and Decision 5: 139-160.

Houthakker, H.S. (1950): “Revealed preference and the utility function”, Economica (N.S.) 17: 159-174. 
Luce, R. D. (1977): “The choice axiom after twenty years”, Journal of Mathematical Psychology 15: 215-233.

Luce, R. D. (1959): Individual Choice Behaviour (New York: Wiley).

Luce, R. D. (1958): “A probabilistic theory of utility”, Econometrica 26: 193-224.

Luce, R. D. and P. Suppes (1965): "Preference, utility and subjective probability”, in R. D. Luce, R. R. Bush and E. Galanter (eds), Handbook of Mathematical Psychology, Vol III (New York: John Wiley and Sons).

McCausland, W. J. (2007): “Random consumer demand”, mimeo, University of Montreal.

McFadden, D. (2005): “Revealed stochastic preference: a synthesis”, Economic Theory 26: 245-264.

Manski, C. F. (1977): “The structure of random utility models”, in G. L. Eberlein, W. Kroeber-Riel, W. Leinfellner and F. Schick (eds) Theory and Decision (Dordrecht: Reidel).

Marschak, J. (1960): “Binary choice constraints and random utility indicators”, in K. J. Arrow, S. Karlin and P. Suppes (eds) Proceedings of a Symposium on Mathematical Methods in the Social Sciences (Stanford: Stanford University Press).

Mas-Colell, A., M.D. Whinston and J. Green (1995): Microeconomic Theory (New York: Oxford University Press).

Nandeibaum, S. (2007): “On the rationalizability of randomized choice”, mimeo, University of Bath.

Quah, J. K-H (2006): “Weak axiomatic demand theory”, Economic Theory 29: 677-699.

Quandt, R. (1956): “A probabilistic theory of consumer behavior”, Quarterly Journal of Economics 70: 507-536.

Richter, M.K. (1966): “Revealed preference theory”, Econometrica 34: 635-645.

Samuelson, P.A. (1948): “Consumption theory in terms of revealed preference”, Economica 15: 243-253.

Samuelson, P.A. (1947): Foundations of Economic Analysis (Cambridge MA: Harvard University Press).

Samuelson, P.A. (1938): “A note on the pure theory of consumer’s behavior”, Economica 5: 61-71.

Sattath, S. and A. Tversky (1976): "Unite and conquer: a multiplicative inequality for choice probability”, Econometrica 44: 79-89.

Sen, A.K. (1971): “Choice functions and revealed preference”, Review of Economic Studies 38: 307317.

Varian, H.R. (1982): “The nonparametric approach to demand analysis”, Econometrica 50: 945-972.

Yellott, J. I., Jr. (1977): “The relationship between Luce’s choice axiom, Thurstone’s theory of comparative judgment, and the double exponential distribution”, Journal of Mathematical Psychology 15: 109-144. 
Figure 1

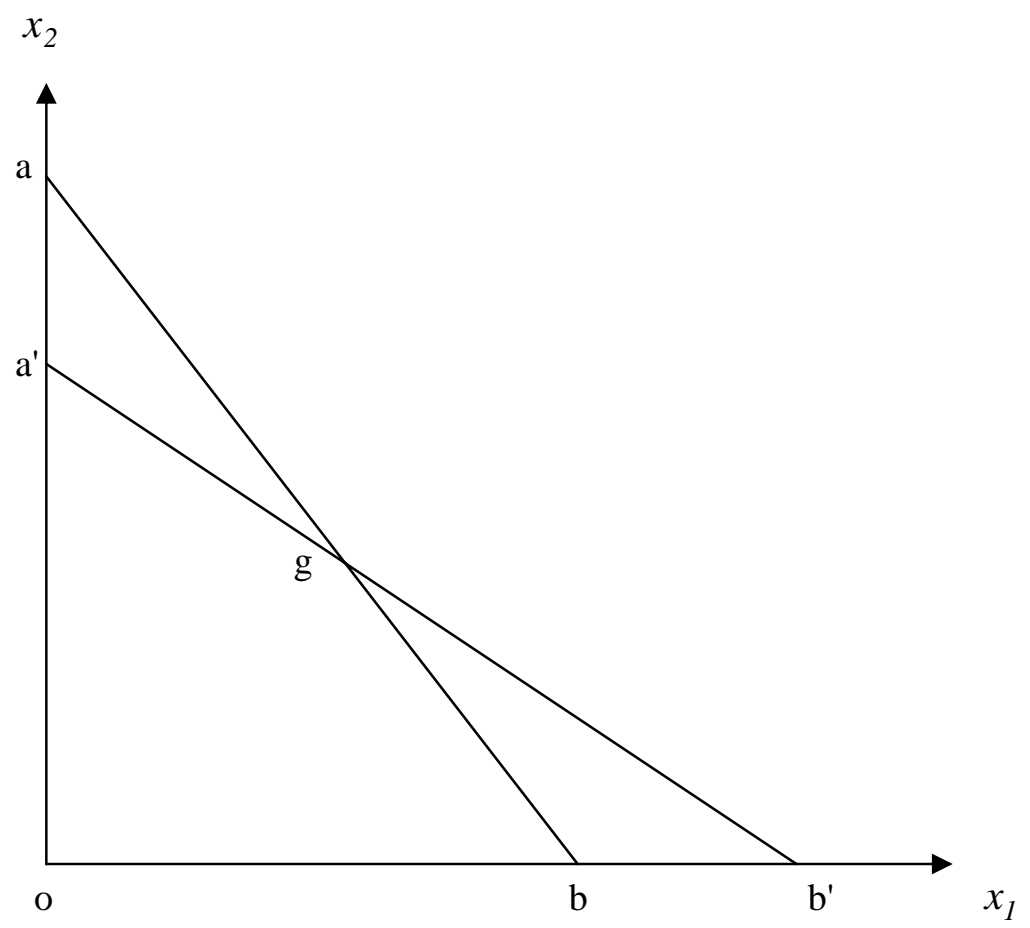

oab is the budget set, $B$, corresponding to the price-wealth situation $(p, W)$.

oa'b' is the budget set, $B^{\prime}$, corresponding to the price-wealth situation $\left(p^{\prime}, W^{\prime}\right)$.

I is the singleton set containing g.

$\mathrm{G}$ is ag excluding the point $\mathrm{g}$.

$\mathrm{H}$ is gb excluding the point $\mathrm{g}$.

$\mathrm{G}^{\prime}$ is gb' excluding the point $\mathrm{g}$.

$\mathrm{H}^{\prime}$ is a'g excluding the point $\mathrm{g}$. 
Figure 2.

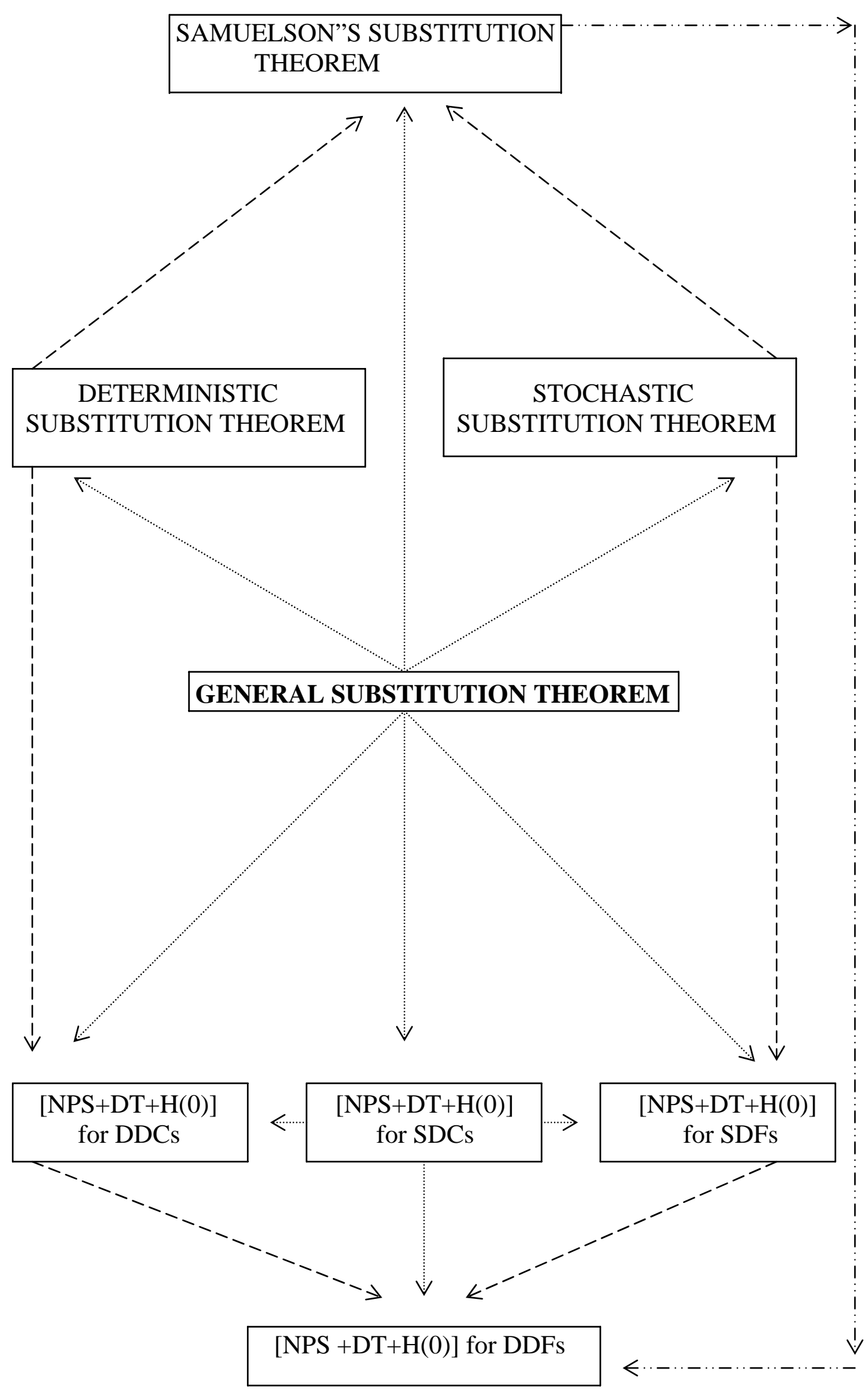

(Note: DT denotes the demand theorem, $\mathrm{H}(0)$ denotes homogeneity of degree 0 in prices and wealth.) 
AWPE DATA SHEET

PD: July 2007

\title{
TI: Revealed preference with stochastic demand correspondence
}

\section{AU: Dasgupta, Indraneel; Pattanaik, Prasanta K.}

AA: Dasgupta: University of Nottingham. Pattanaik: University of California.

SR: $\quad$ University of Nottingham, Discussion Papers in Economics, Discussion Paper No. 0706; Discussion Paper Coordinator, School of Economics, University of Nottingham, University Park, Nottingham, NG7 2RD, UK.

http://www.nottingham.ac.uk/ lezec/research/dp/

PG: 20

PR: Nil

JE: $\quad$ D11

KE: Stochastic demand correspondence, weak axiom of revealed preference, weak axiom of stochastic revealed preference, general substitution theorem, demand theorem.

\begin{abstract}
AB:
We unify and expand the theory of consumer's behavior, based on Samuelson's Weak Axiom of Revealed Preference, to permit simultaneously both random choice and non-singleton choice sets. We provide a consistency postulate for demand behavior when such behavior is represented in terms of a stochastic demand correspondence. When the consumer spends her entire wealth, our rationality postulate is equivalent to a condition we term stochastic substitutability. This equivalence generates: (i) Samuelson's Substitution Theorem, (ii) the central result in Bandyopadhyay, Dasgupta and Pattanaik (2004) and (iii) a version pertinent to deterministic demand correspondences (which independently yields Samuelson's Substitution Theorem), as alternative special cases. Relevant versions of the non-positivity of the own substitution effect, the demand theorem and homogeneity of degree zero in prices and wealth for the consumer's demand behavior, also follow as corollaries in every case.
\end{abstract}

